\title{
Mathematische Schneeschmelze
}

\author{
Rainer Kaenders und Ysette Weiss
}

\begin{abstract}
Seit mehr als einer Dekade fehlen einem Großteil der Studierenden bei Studienbeginn viele mathematische Grundkenntnisse und -fertigkeiten sowie ein konzeptuelles Verständnis mathematischer Inhalte. Gleichzeitig fallen die Abiturnoten immer besser aus und die Abiturientenquote steigt unaufhörlich. Mit Bologna und mit der Einführung der sogenannten Bildungsstandards hat der Staat deutschen Schulen, Hochschulen und Studienseminaren die Sprache der Kompetenzen als universelle Sprache für die Beschreibung, Planung, Testung und Weiterentwicklung von Lehr- und Lernprozessen gesetzlich vorgeschrieben. Im Mathematikunterricht wurden dabei viele klassische Inhalte gestrichen. Die Umstellung auf Output-Orientierung führte zum Bruch mit einer ehemals international beachteten Bildungstradition der Aufklärung.
\end{abstract}

In dem Brandbrief „,von mehr als 130 Professoren und Lehrkräften" (Tagesspiegel vom 22.3.2017, Mathematikunterricht und Kompetenzorientierung - ein offener Brief [3]) wird explizit die Kompetenzorientierung im Mathematikunterricht für den inzwischen unbestrittenen Niedergang der elementaren mathematischen Fähigkeiten eines Großteils der Studierenden bei Studienbeginn verantwortlich gemacht. In ihrer Replik antworten 50 Angehörige unserer mathematikdidaktischen Zunft hierauf (siehe [4]):

Es gibt keinen Zweifel, dass Studienanfänger(innen) über substantielles mathematisches Basiswissen (wie z. B. auch in den im Brief genannten Bereichen Bruchrechnung, binomische Formeln, Termumformungen, Elementargeometrie oder Trigonometrie) verfügen müssen. Sie müssen aber dieses Wissen auch verständig anwenden und hiermit innermathematische Probleme sowie Probleme der realen Welt lösen können - das ist knapp gefasst die Grundidee der Kompetenzorientierung.

Was hat es mit dem Streit um die Kompetenzorientierung auf sich? Ist das nicht eigentlich ein neues Wort für so etwas wie Lernziele? Gab es das nicht immer schon? Und wer kann etwas dagegen haben, dass die Schulen und Universitäten zumindest als Ziel formulieren, kompetente Absolventen zu entlassen? Kann jemand nicht wollen, dass sie dieses Wissen auch verständig anwenden und hiermit „innermathematische Probleme“ sowie Probleme der realen Welt lösen können? Haben sich da die Praktiker und Theoretiker der mathematischen Lehre auf ein Feindbild eingeschossen, das gar nicht als solches taugt? Ist es nicht gut, wenn die Anforderungen vereinheitlicht werden, sodass sie auch systematisch vermittelt werden können? Weiten Teilen einer allgemeinen mathematischen
Öffentlichkeit erscheint dieser kritische Diskurs womöglich als Wortklauberei.

Die Einführung der Kompetenzorientierung als universelles und gesetzlich verordnetes Paradigma für die Beschreibung und Gestaltung von Lernprozessen verändert allerdings sehr konkret die mathematische Kultur in Lehre und Forschung. Für Lehrkräfte der Mathematik in Schule und Hochschule ist es von existenzieller Bedeutung, diese Entwicklungen genauer zu verstehen und sich auf einen geisteswissenschaftlichen Diskurs mit pragmatischen Folgen einzulassen, der grundsätzlich nicht durch Empirie geklärt werden kann und sich auch nur teilweise innerhalb der mathematischen Kultur abspielt. Wir haben es bei der Kompetenzorientierung mit einer fundamentalen Veränderung unseres Verständnisses vom Lernen zu tun. Geht es darum, zu lernen, etwas zu verstehen; oder geht es darum, andere davon zu überzeugen, etwas verstanden zu haben?

Das begriffliche System der Kompetenzorientierung stammt aus der angewandten Psychologie (Gelhard 2012). Dort hat es lange vor allem zur Selektion und Anpassung von Arbeitskräften gedient, die speziell definierten psychologischen Anforderungen am Arbeitsplatz, wie Geduld, Genauigkeit, Schnelligkeit, etc. genügen sollen. Obwohl die Kompetenzorientierung in Bezug auf Unterricht auf Initiative der OECD (vgl. Weinert 2002, S. 27) von vorrangig quantitativ empirisch arbeitenden pädagogischen Psychologen und Didaktikern vorangetrieben wurde, fehlt bis heute ein empirisch zweifelsfreier Nachweis, dass sich die gegenwärtig staatlich umgesetzte Kompetenzorientierung positiv auf die Kenntnisse und Fertigkeiten von Abiturienten beziehungsweise Studienanfängern ausgewirkt hat. Kann so ein Nachweis überhaupt geführt werden? Das Problem ist: Der Kompetenzbegriff ist in seiner Anlage prinzipiell nicht geeignet, Fachsystematik begrifflich abzubilden. Dies kann erst wirklich deutlich gemacht werden, wenn man einen konkreten 
fachlichen Gegenstand betrachtet. Die von den 5o Didaktikerinnen und Didaktikern skizzierte Grundidee ist eben nicht das, was die Kompetenzorientierung ausmacht, sondern sie ist nur eines der Heilsversprechen, mit denen die Kompetenzorientierung daherkommt. Am Beispiel einer in den Mitteilungen der DMV schon geführten Auseinandersetzung werden wir dies näher erläutern.

\section{Momentane Änderungsrate der Schneehöhe}

Franz Lemmermeyer stellte in seinem Beitrag in den Mitteilungen der DMV 24-3 (2016) anhand einer Abituraufgabe aus Baden-Württemberg die Problematik der zentralen Abiturprüfungen und der Struktur des gegenwärtigen Mathematikunterrichts sehr fundiert dar: Der mathematische Anspruch der Aufgaben ist vorwiegend Mittelstufenniveau - wo Begriffe der Oberstufe in die Aufgabenstellungen eingehen, sind die Lösungswege stark schematisiert und die Funktionstypen und Formeln vereinfachte Spezialfälle. Die von komplexeren Inhalten entkernten („entrümpelten“) Kerncurricula gestatten auch kaum die Verwendung komplexerer Terme und Funktionen. Die häufig anzutreffende Auffassung, dass der niedrige mathematische Anspruch durch Einbettung in komplexe Anwendungszusammenhänge gehoben würde, ist ein auf die Situation unzutreffender Allgemeinplatz, der von der konkreten Scheinmodellierung ablenkt.

In seinem Leserbrief $(24-4,2016)$ schreibt Werner Blum hierzu, dass er „die von Lemmermeyer inkriminierten Abituraufgaben nicht global verteidigen" wolle. Doch beharrt er darauf, dass das Lösen dieser beiden Aufgaben „über bloßes Lesen weit hinausgehende Anforderungen“, beispielsweise ein Verständnis der „momentanen Änderungsrate der Schneehöhe" stelle.

Schauen wir uns dazu noch einmal den von Lemmermeyer besprochenen Aufgabenteil zur momentanen Änderungsrate der Schneehöhe (A 2.1) an:

In einem Skigebiet beträgt die Schneehöhe um 10:0o Uhr an einer Messstelle $150 \mathrm{~cm}$. Die momentane Änderungsrate dieser Schneehöhe wird beschrieben durch die Funktion $s$ mit

$$
s(t)=16 e^{(-0,5 t)}-14 e^{(-t)}-2 ; \quad 0 \leq t \leq 12
$$

( $t$ in Stunden nach $10.00 \mathrm{Uhr}, s(t)$ in Zentimetern pro Stunde).

Im Anschluss sollen dann im Wesentlichen die maximale lokale Änderungsrate (a) und ein integralfreier Funktionsterm, der die Schneehöhe zum Zeitpunkt t beschreibt (b), jeweils bestimmt werden, bevor im Aufgabenteil (c) die lokale Änderungsrate noch einmal - durch den ab einem bestimmten Zeitpunkt stattfindenden Einsatz von Schneekanonen - mit einem konstanten Term verändert wird und in der variierten Situation wiederum auf die Schneehöhe geschlossen werden soll.

Zunächst geht es hier um Skigebiete und Schneehöhen. Schüler, die sich diesen Kontext vor Augen führen, könnten jetzt eventuell an eine Schneelandschaft mit geschwungenen Schneehöhen denken. Das erinnert an Funktionsgraphen. Doch Vorsicht, hier lauert ein Distraktor es geht um die Abhängigkeit der Schneehöhe von der Zeit. Der Versuch, diese nun erkannte Situation zu verstehen, lässt an einen zusammensackenden Schneehaufen denken, auf den es hin und wieder schneit. Was bedeutet hier momentane Änderungsrate im Kontext schmelzender und sich auftürmender Schneekristalle? Was genau verändert sich hier und was heißt dann momentan? Und wieso soll man die momentane Änderungsrate einfacher messen können als die Schneehöhe selbst? Das alles ist nicht einfach.

Ein erster, wohl naiver Versuch hierüber Hypothesen wie „bei gleichmäßigem Schneefall ist die lokale Änderungsrate konstant" o. ä. aufzustellen, wäre allerdings nicht klug - nicht, weil der Sachverhalt eigentlich komplizierter ist - sondern, weil man sich hierdurch von der erfolgreichen Bearbeitung der Abituraufgabe nur entfernen würde. Nun erweist es sich als ein Vorteil, dass im Vorfeld beim Training gelernt wurde: Lese „momentane Änderungsrate" und verstehe „Ableitung“. Diese momentane Änderungsrate der Schneehöhe wird beschrieben durch die Funktion $s$ mit $s(t)=16 e^{(-0,5 t)}-14 e^{(-t)}-2 ; 0 \leq t \leq 12$. Eingeweihte wissen, dass das (a) nicht so ist und (b) daran liegt, dass die Schüler in Ermangelung weiterer mathematischer Fertigkeiten nur noch mit ganzrationalen Funktionen oder Linearkombinationen von Termen der Form e-Funktion hoch linearer Term konfrontiert werden dürfen. Bei letzteren handelt es sich um sogenannte D-Funktionen (Carl-Peter Fitting), da in NRW solche Entwicklungen immer aus Düsseldorf kommen. 2007 wurden dort übrigens auch Primzahl, Primfaktorzerlegung, Höhen- und Kathetensatz, Sinus- und Kosinussatz, Logarithmengesetze und einiges mehr und dann 2014 noch die Quotientenregel, die gebrochen-rationalen Funktionen und die Kosinusfunktion ,entrümpelt“.

D-Funktionen können die Schüler durch ausgiebiges Üben noch differenzieren (und integrieren), indem sie bei Potenzfunktionen den Exponenten davorschreiben und um 1 erniedrigen und bei e-Funktion hoch $l i$ neare Funktion, die Steigung der linearen Funktion einfach davorschreiben. Das kann man sich merken. Dieses Herrschaftswissen zum Differenzieren und Integrieren von D-Funktionen übrigens verhindert möglicherweise, dass auch Neuntklässler die Abituraufgabe schon lösen könnten, wie das in der Biologie schon lange der Fall ist (Jahnke \& Klein, 2012). Für Österreich haben Bandelt und Kühnel $(2016,2017)$ nachgewiesen, dass auch die mathematische Reifeprüfung schon mit dem Stoff des 9. und 10. Schuljahres bestanden werden kann - sogar mit der Note befriedigend.

Kompetenzen - ein Werkzeug der angewandten Psychologie

Die von Lemmermeyer besprochene Aufgabe ist exemplarisch. Dazu muss man wissen, dass die verwendeten Imperative lange nicht einfordern, was sie suggerieren 
oder was die Sachlage gebieten würde, sondern dass es sich hier um sogenannte Operatoren handelt, wie wir unten näher erläutern werden. Hierdurch und durch die anwendungsbezogen wirkenden Kontexte sehen die Aufgaben anspruchsvoll aus und können dem ersten Blick von Bildungsökonomen, Eltern, Politikern (und vielleicht auch manchem mathematisch gebildeten Hochschulangehörigen) standhalten. Es handelt sich aber um standardisierte Aufgabentypen, die immer nach denselben zigfach eintrainierten Schemata (siehe Jahnke 2016) abgearbeitet werden. Die wohlfeilen kompetenzorientierten Formulierungen der Bildungsstandards geben keine Orientierung bezüglich der Inhalte.

In Wirklichkeit sind die zentralen Prüfungen - und nicht die Kernlehrpläne - zum geheimen Lehrplan geworden und damit im Übrigen auch nicht mehr Gegenstand demokratischer Willensbildung. Eingeführt unter dem Vorwand, die Kultur fester Lösungsschemata abzulösen, sind genau diese Lösungsschemata unter der Kompetenzorientierung zum zentralen Unterrichtsgegenstand geworden. Die eigentliche Schwierigkeit der Aufgaben besteht dabei nicht so sehr in der Mathematik, vielmehr gilt es, diese von ihren textlichen Distraktoren zu befreien und herauszufinden, welches der eintrainierten Schemata aktiviert und nachgeahmt werden muss. Der (gymnasiale) Mathematikunterricht ist in der Krise!

Die Ursache für diese Entwicklung sehen immer mehr Fachdidaktiker und Erziehungswissenschaftler in der Kompetenzorientierung. Sie dringt tiefer und tiefer in alle Bereiche des deutschen Bildungssystems ein. Auf Betreiben der Hochschulrektorenkonferenz wird sie auch an Universitäten noch radikalere Formen annehmen, als sie es seit Einführung des Bachelor-Master-Systems schon tut. Das Ziel der Politik ist es, den Output bei Tests wie PISA oder TIMSS zu verbessern. Die Kompetenzorientierung verspricht unter anderem, dies zu können und Bildung zu einem beherrschbaren System zu machen:

Bildungsstandards mit ihrem Bezug zu Schülerkompetenzen sind explizit so formuliert, dass sie mit Hilfe entsprechender Aufgaben bzw. Tests überprüft werden können. Diese Messbarkeit zeichnet sie national und international aus, und bei aller Bescheidenheit ist es diese Eigenschaft, die es erlaubt, zu bestimmten Zeitpunkten festzustellen, ob und in welchem Ausmaß Schülerinnen und Schüler für das weitere Leben adäquat gerüstet sind bzw. ob Optimierungsbedarf besteht. [Blum et al. 2006, S. 9]

Die Kompetenzorientierung entspricht in ihrer ökonomischen Sprache den Vorgaben der Wirtschaftsorganisation OECD, die Bildung und Menschen ökonomisch als $\mathrm{Hu}$ mankapital interpretiert. Bildungswissenschaftler wie Franz Weinert (2002, S. 26) und viele andere haben dies dann umgesetzt.

In der vorgestellten Aufgabe sollen die Schüler Modellierungskompetenz unter Beweis stellen. Nie geht es, wie wir auch hier sehen, direkt um Inhalte - die sind in dieser
Sprache eben nicht formulierbar. Die häufig genannten Kompetenzen „zu beweisen“ oder „zu argumentieren“ (Blum 2016) betreffen texthaltige Aufgaben und ihre ordnungsgemäß mit Schlüsselworten versehenen texthaltigen Lösungen, wie sie die Aufgabensteller erwarten.

„Es soll mit Wolle gestrickt werden“ (Blum 2016). Doch nie wird gesagt, um welche Wolle es sich handelt. Es wird auch nicht klar, welche Wolle denn die beste Wolle zum Strickenlernen ist. Inhalte werden nur beispielhaft und nicht exemplarisch behandelt - für letzteres bräuchte man ein Verständnis der Rolle der Inhalte in der Fachkultur.

Wie kann man einen Zahlbegriff ohne Primzahlen entwickeln, wie geometrische Anschauung ohne Elementargeometrie, wie reelle Zahlen ohne Irrationalität, wie Trigonometrie ohne Einheitskreis und Kosinusfunktion, wie das Skalarprodukt ohne Kosinussatz, wie analytische Geometrie ohne Geometrie, wie lineare Algebra ohne Linearität, wie Kombinatorik ohne Zählverfahren, wie Binomialverteilung ohne den binomischen Lehrsatz, wie die Differential- und Integralrechnung ohne einen (wenn auch nur anschaulich tauglichen) Grenzwertbegriff, wie mathematische Anwendungen ohne anwendbare Mathematik, wie mathematisches Modellieren ohne mathematische Modelle, wie mathematisches Denken ohne Begründungen und Beweise?

Die Kompetenzorientierung macht all das derzeit in Deutschland möglich! Es sollen ja nicht Inhalte verstanden, sondern Kompetenzen erworben und Erträge schulischen Lernens (Weinert 2002, S. 28) erzielt werden.

Die OECD hat in diesem Zusammenhang mehrfach vorgeschlagen, den vieldeutigen Leistungsbegriff generell durch das Konzept der Kompetenz zu ersetzen (vgl. [...]). Dabei versteht man unter Kompetenzen die bei Individuen verfügbaren oder durch sie erlernbaren kognitiven Fähigkeiten und Fertigkeiten, um bestimmte Probleme zu lösen sowie die damit verbundenen motivationalen, volitionalen und sozialen Bereitschaften und Fähigkeiten, um die Problemlösungen in variablen Situationen erfolgreich und verantwortungsvoll nutzen zu können. (Weinert 2002, S. 27-28)

Der auf dieser Definition von Franz Weinert beruhende Kompetenzbegriff, das Credo der Testindustrie, wurde zum zentralen Begriff des Umbaus unseres gesamten Bildungssystems. Er entwickelte sich vom psychologischen Selektionsinstrument zum Leitbegriff für die industrielle Qualitätskontrolle der Lieferanten von Humankapital für Wirtschaftssysteme - wie die OECD sie seit Jahrzehnten regelmäßig durchführt.

Kompetenzen sind eben, man kann es nicht oft genug betonen, ein psychologisches Instrument. Modellieren, Zusammenarbeiten, Argumentieren und sogar moralische Kompetenzen (Weinert 2002, S. 28), etc. werden zu kontextfreien Problemlösetätigkeiten erhoben. Da sie keinem Kontext gerecht werden müssen, werden sie zu beobachtbaren und messbaren psychologischen Kategorien. Man 


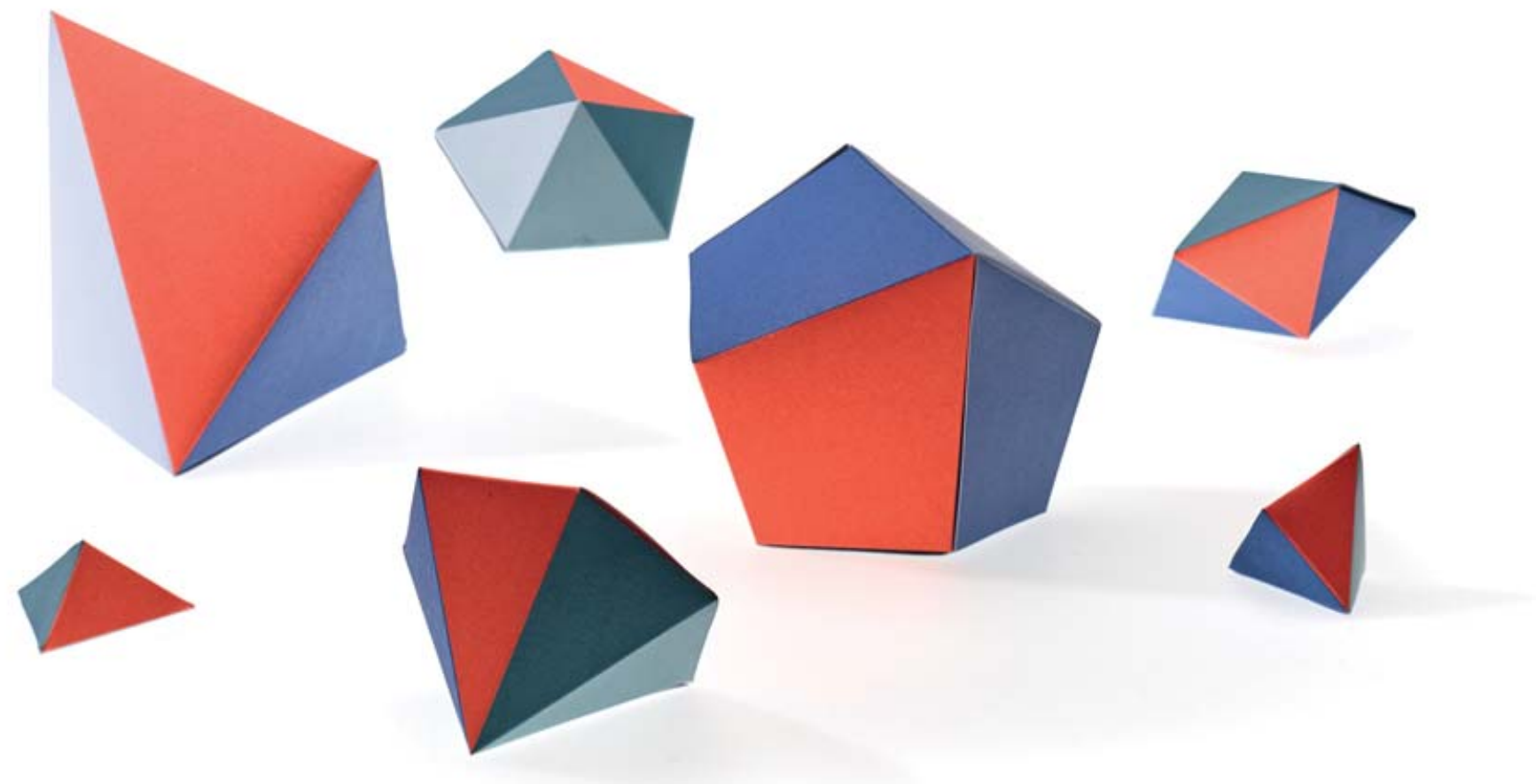

(Abbildung: Christoph Eyrich)

modelliert beispielsweise, wenn man das Vokabular eines didaktischen Modellierungskreislaufs verwendet. Neben der psychologischen Selektions- und Anpassungsfunktion haben die Kompetenzen noch einen weiteren Hintergrund, der schon bei Weinert (2002, S. 18) angedeutet wird, wenn er im Namen der „leistungsorientiert eingestellten Pädagogen und Bürger" Leistung als eine „Manifestation eines menschlichen Grundbedürfnisses, eine Möglichkeit der individuellen Selbstverwirklichung durch Erfahrung eigener Selbstwirksamkeit" identifiziert. Dabei hält Weinert intrinsische Motivation für einen der Faktoren, dessen „Wirksamkeit in einem Teil der Literatur bis heute überschätzt wird" (2002, S. 25). Schon in den 8 oer Jahren haben die Psychologen Deci und Ryan (2000) eine in den Fachdidaktiken weit rezipierte „Selbstbestimmungstheorie" entwickelt, welche die Verinnerlichung extrinsischer Motivation über das Erleben von Kompetenz, Autonomie und sozialer Eingebundenheit beschreibt.

Die Output-Orientierung bringt die Selektions- und die Motivationsdimension von Kompetenz zusammen, indem die Operationalisierungen (vgl. Baumert et al. 2000, S. 11) solcher Motivationstheorien für den Nachweis „motivationaler Präferenzen“ der Schüler als Teil eines allgemeinen Kompetenzbegriffs eingesetzt werden. Diese motivationalen Präferenzen werden folgerichtig auch innerhalb der PISA-Studie erhoben (Baumert et al. 200o).

Pointiert zusammengefasst kann man sagen: Die staatlich verordnete Kompetenzorientierung ist eine Methode, mittels der durch die Verinnerlichung extrinsischer Motivationen die Verwendbarmachung von Humankapital zur Förderung der wirtschaftlichen Entwicklung (vgl. Weinert,
S. 26) anstrebt wird, was - wie wir jetzt in der Mathematik wissen - noch nicht einmal funktioniert.

In der Bildungstradition, die mit Platon ihren Anfang nahm und mit der Aufklärung und dem Neuhumanismus zur Grundlage des europäischen Bildungsverständnisses wurde, sind es die Gegenstände, Phänomene und geistigen Schöpfungen dieser Welt selbst, die uns mit ihrem Eros (Platon) in ihren Bann ziehen und das Wahre, das Gute und das Schöne suchen lassen (Dörpinghaus 2009). Das Kantsche Sapere aude! ruft dazu auf, aus eigener Motivation zu denken. Das psychologische Instrument der Kompetenz dagegen erzielt seine Wirkung, indem es einfach subjektiv erlebt wird und damit eine emotionale Grundlage für die dann folgende Fremdbehandlung liefert, ohne notwendig einem Gegenstand, einem Phänomen oder einer geistigen Schöpfung gerecht werden zu müssen.

In unserem Beispiel reicht es eben, das Gefühl zu haben, etwas Sinnvolles über Schneehöhen sagen zu können. Als Kompetenz kann man daher - scheinbar über alle Fächer hinweg - universelle Fertigkeiten definieren, die keine fachspezifischen Grundlagen benötigen. Mit ihnen können die in den einzelnen Fachkulturen etablierten wichtigen Fertigkeiten imitiert werden, solange sie von den Testerinnen und Testern als Kompetenz wahrgenommen werden. Entsprechende Begriffe und ihr Verständnis sowie der Nachweis, dass dieses Verständnis vorhanden sei, werden aus dem Kontext genommen und über psychologische Tests operationalisiert. Dies zeitigt dann fachlich die wunderlichsten Folgen. Eine fundierte und geistreich geschriebene Lektüre zu diesen Folgen ist (Klein 2016). 
Operatoren als totalitäre Sprachregelung

Schon in der Elementarmathematik fragen sich Lehrer und Schüler beispielsweise, ob eine Argumentation als Beweis gelten kann, oder ob eine Berechnung zu einer logisch belastbaren Aussage führt, ob eine Zeichnung einen mathematischen Sachverhalt wiedergibt, ob und inwieweit eine Herleitung als gelungen betrachtet werden kann, welche Eigenschaft eines Objekts zur Definition erhoben werden sollte. An solchen und anderen derartigen Fragen vollzieht sich mathematische Entwicklung über Diskurs. Mathematische Begriffe zeichnen sich dabei jedoch überraschenderweise durch eine intrinsische oder platonische Unvermeidlichkeit aus. Hier und da ist es allerdings auch sinnvoll über die Einführung von Begrifflichkeiten zu diskutieren. Soll es unter ebenen Polygonen ein Zweieck geben? Vom Standpunkt der Innenwinkelsumme ja, aus Sicht der Triangulierbarkeit wiederum nicht. In derartigen offenen Diskursen unterscheidet sich Mathematik zum Beispiel von der an gegebenen Gesetzen orientierten Jurisprudenz.

So wie Facebook den Begriff befreundet neu definiert hat, so nehmen die Kompetenzapologetinnen und -apologeten mit ihren Operatoren den Menschen in der Mathematik nun seit Jahren die eigene Sprache mit ihren offenen kreativen Möglichkeiten, ohne dass die meisten sich dessen bewusst wären - geschweige denn, dass sie sich darüber aufregten. Dies muss man wissen, wenn man die oft anspruchsvoll klingenden Formulierungen der Abituraufgaben verstehen will. Die Verben angeben, nennen, berechnen, beschreiben, erstellen, darstellen, skizzieren, zeichnen, graphisch darstellen, bestimmen, ermitteln, entscheiden, erklären, herleiten, interpretieren, untersuchen, prüfen, vergleichen, zeigen, nachweisen, beurteilen, beweisen und widerlegen wurden in der Mathematik - sei es in der Schule oder der Universität - immer so verstanden, wie das im Dialog zwischen Mathematiktreibenden, zwischen Lehrern und Schülern, als sinnvoll erfahren wurde. In solchem Verständnis zeigte sich eben gerade mathematische Fähigkeit, wobei eben auch das Ringen um eine Deutung zu Entwicklung und interessanten Einsichten führte.

Nun sind die Operatoren als unmissverständliche Handlungsanweisungen, als Befehle, fixiert. In den meisten Bundesländern (für NRW siehe [1]) hat der Staat im Rahmen der sogenannten Standardsicherung (Was muss hier eigentlich wogegen gesichert werden?) jedes einzelne der obigen Verben in drei Anforderungsbereichen juristisch hieb- und stichfest festgelegt. Nicht selten führt diese mathematische Jurisprudenz zu Unsinn. Warum wir das so einschätzen? Nun ja, wir können das beurteilen; mal schauen ...

$\mathrm{Zu}$ Sachverhalten ein selbstständiges Urteil unter Verwendung von Fachwissen und Fachmethoden formulieren und begründen.

Nehmen wir den Operator angeben, nennen, dann finden wir:
Objekte, Sachverhalte, Begriffe, Daten ohne nähere Erläuterungen, Begründungen und ohne Darstellung von Lösungsansätzen oder Lösungswegen aufzählen.

Das klingt harmlos und schlicht - wie die berühmte (und wohl nicht mehr geltende) zentrale Dienstvorschrift der Bundeswehr ZDv 10/5 Leben im Felde: „Ab einer Wassertiefe von 1,20 $\mathrm{m}$ hat der Soldat selbständig Schwimmbewegungen aufzunehmen. Die Grußpflicht entfällt." Doch die Operatoren sind nicht nur wunderliche Formulierungen von Offensichtlichem; sie sind eben auch gefährlich, denn sie entbinden den Lernenden davon, über die Sinnhaftigkeit seiner Antwort nachzudenken. Der Operator ermitteln etwa kommt unschuldig daher mit: Zusammenhänge bzw. Lösungswege aufzeigen, das Vorgehen darstellen und die Ergebnisse formulieren. In der Praxis führt dies aber häufig zu nichts anderem, als dem von Lemmermeyer monierten Unterricht als das Einüben des Übersetzens von Modellierungsaufgaben in Taschenrechnerbefehle. Ähnlich verhält es sich mit anderen Operatoren.

Auch in der universitären Lehrerbildung - und sicher auch in anderen Studiengängen - fordern die Studierenden immer häufiger den Gebrauch dieser staatlichen Operatoren („schöne klare Imperative“, Zitat eines Schülers) ein, bei denen man sich keine Gedanken mehr darüber machen muss, ob die jeweilige Antwort im Kontext sinnvoll und zufriedenstellend ist. Formuliert man in einer Klausur etwa, dass die Studierenden etwas angeben sollen, dann muss man sich nicht wundern, wenn die Studierenden „Objekte, Sachverhalte, Begriffe, Daten ohne nähere Erläuterungen und Begründungen ohne Darstellung von Lösungsansätzen oder Lösungswegen aufzählen“, auch wenn das in der jeweiligen Situation absurd wirkt. In der Klausureinsicht folgt der Verweis, die Aufgabe sei mit der Aufzählung einiger Stichworte korrekt bearbeitet, weil ja der Operator angeben erfüllt sei. Sind wir die einzigen Kollegen, die derartiges erleben?

\section{Modellierung und Anwendung}

Gilbert Greefrath, Matthias Ludwig und Stephan Siller sprechen in ihrer Reaktion auf Lemmermeyers kritische Analyse (Greefrath et al. 2016) davon, dass sich bei der Lektüre der Eindruck aufdränge, „... dass Mathematik hier nicht als allgemeinbildendes Unterrichtsfach, sondern einseitig innermathematisch verstanden" werde. Was „allgemeinbildend“ bedeutet, erläutern sie anhand der Winterschen Grunderfahrungen. Doch wer könnte eigentlich gegen die Winterschen Grunderfahrungen sein? Hier liegt nicht das Problem. Weiter heißt es in ihrer Replik: „Alle drei Grunderfahrungen sind gleichbedeutend und auch die Grundlage für aktuelle Lehrpläne und Bildungsstandards." Das allerdings ist falsch.

Beispielsweise hat die Landesregierung in NRW diese Grunderfahrungen ohne weiteren Kommentar nur scheinbar übernommen; sie wurden kompetenzifiziert. Im Lehrplannavigator S II Gymnasiale Oberstufe Mathematik 


\section{Grunderfahrung nach Heinrich Winter}

(G1) „Erscheinungen der Welt um uns, die uns alle angehen oder angehen sollten, aus Natur, Gesellschaft und Kultur, in einer spezifischen Art wahrzunehmen und zu verstehen,"

(G2) „mathematische Gegenstände und Sachverhalte, repräsentiert in Sprache, Symbolen, Bildern und Formeln, als geistige Schöpfungen, als eine deduktiv geordnete Welt eigener Art kennen zu lernen und zu begreifen und“

$\left(\mathrm{G}_{3}\right)$,in der Auseinandersetzung mit Aufgaben Problemlösefähigkeiten, die über die Mathematik hinaus gehen, (heuristische Fähigkeiten) zu erwerben."
Kernlehrplan NRW

Schülerinnen und Schüler sollen

...technische, natürliche, soziale und kulturelle Erscheinungen und Vorgänge mithilfe der Mathematik wahrnehmen, verstehen, beurteilen und beeinflussen (Mathematik als Anwendung).

...mathematische Gegenstände und Sachverhalte, repräsentiert in Sprache, Symbolen und Bildern, als geistige Schöpfungen, als eine deduktiv geordnete Welt eigener Art erkennen und weiterentwickeln (Mathematik als Struktur),

... in der Auseinandersetzung mit mathematischen Fragestellungen Kreativität und Problemlösefähigkeit, die über die Mathematik hinausgehen, erwerben und einsetzen (Mathematik als individuelle und kreative Tätigkeit).
[2] finden wir zur Einleitung in den Kernlehrplan die Aufgaben und Ziele des Faches etwas, das bei oberflächlicher Lektüre aussieht wie die Winterschen Grunderfahrungen, doch bei genauem Hinschauen etwas ganz anderes meint.

Beispielsweise ist wahrnehmen und verstehen etwas anderes als wahrnehmen, verstehen, beurteilen und beeinflussen. Können und sollen Schülerinnen und Schüler technische, natürliche, soziale und kulturelle Erscheinungen und Vorgänge beurteilen und beeinflussen? Muss man dazu nicht über Legitimation, Erfahrung und Kompetenz - im eigentlichen Wortsinn - verfügen? Dass die Landesregierung mathematische Gegenstände und Sachverhalte von den Schülern weiterentwickeln lassen will, die zuvor als Muster erkannt und nicht als Mathematik kennengelernt und begriffen wurden, erschließt sich nur dem, der an solch großspurige Prosa von Bildungsökonomen gewöhnt ist.

Vor allem aber bezieht sich die Problemlösefähigkeit bei Winter auf die Kultur des Aufgabenlösens in der Mathematik, wie sie etwa Polya beschrieben hat, und auf Modellierung in wirklichen Kontexten. Die Problemlösefähigkeit im Kernlehrplan hingegen bezieht sich auf einen der umstrittensten Begriffe der Kompetenzorientierung (siehe beispielsweise Wiechmann 2013 oder Gelhard 2012). Sie hat so gut wie nichts mit dem Lösen mathematisch anspruchsvoller Aufgaben und Problemstellungen zu tun, wie wir nicht nur bei den Abituraufgaben seit vielen Jahren sehen. Diese kompetenzorienterten Problemlösefähigkeiten sollen konsequenterweise nicht nur zur Verfügung stehen, sondern einsetzbar sein und das in der Regel nicht vom Lernenden selbst. Letzterer soll allerdings dazu bereit sein, sich einsetzen zu lassen.

Aus der Kompetenzperspektive wird Problemlösefähigkeit häufig mit der Anwendung von Mathematik und dem Modellieren mit Mathematik in Verbindung gebracht. Beides hatte eine wichtige, aber keine universelle Bedeutung für den Mathematikunterricht vor dem PISA-Schock. Für die Anwendung von Mathematik waren früher nicht zuletzt auch die Physik und noch viele andere - mittlerweile demathematisierte - Fächer verantwortlich: Informatik, Chemie, Biologie, Erdkunde, Ökonomie, Sachkunde, ...

Aber auch im deutschen Mathematikunterricht selbst haben anwenden und modellieren eine lange Tradition, und es sind über Jahrhunderte hinweg viele gelungene Beispiele dafür entwickelt worden. Unter diesen gibt es auch zeitgenössische motivierende Kontexte und darunter wiederum gibt es auch welche von den Kollegen Blum, Greefrath, Ludwig und Siller. Doch wirkliches Anwenden und erst recht Modellieren sind schwer. Sie werden nur von besonders engagierten Lehrern mit fachlichem Bezug zu den Kontexten beherrscht. Gleichwohl wird das Modellieren in den Kernlehrplänen gefordert; das durchaus sinnvolle klassische Abfragen von Bedeutungen mathematischer Begriffe in metaphorischen Kontexten gilt als rückständig.

Von der besprochenen Abituraufgabe als Beispiel für Modellierungsaufgaben distanzieren sich die drei Autoren in ihrem Leserbrief auch, indem sie die derzeit gängigen mathematikdidaktischen Merkmale und Klassifikationen für gute Modellierungsaufgaben zitieren. Sie halten die Betrachtung von Abituraufgaben zur Analyse des Mathematikunterrichts generell für ungeeignet, stellen aber nicht klar, dass sich Modellierungsaufgaben überhaupt nicht für Abituraufgaben und andere zentrale Tests eignen.

Das Problem sind nicht Modellierungsaufgaben, sondern solche Pseudomodellierungsaufgaben, wie die gängigen Abituraufgaben. Der Grund für die Popularität letzterer und damit auch der modellierenden Mathematikdidaktik liegt in der Bedeutung dieser Aufgaben beim Vortäuschen eines nicht vorhandenen Niveaus und dem Imitieren komplexer mathematischer Aktivitäten, wie das durch PISA und andere Output-Untersuchungen gefördert wurde und wird. Pseudomodellierungsaufgaben beziehen ihre Bedeutung für den Schulunterricht eben 
nicht aus fachübergreifenden Ansätzen und damit einer bewussten Mathematisierung naturwissenschaftlicher Themen, sondern aus dem Vorgaukeln universeller Modellierungsfähigkeiten, die nicht erst exemplarisch in Anwendungskontexten unter Anstrengung und längerem Zeitaufwand entwickelt werden müssen. Zur Aufrechterhaltung dieser absurden Praxis wird die fachbezogene inhaltliche Diskussion über die Chimäre allgemeingültiger universeller Kompetenzen ideologisiert und nur noch mit psychologischen Instrumenten geführt.

\section{Die Krise ist manifest}

Die mathematischen Defizite der Studierenden an den Universitäten sind längst nicht mehr durch Brücken- und Vorkurse zu kompensieren. Überspitzt könnte man sagen, dass nach der bis heute folgenreichen New MathBewegung mit ihrer anti-didaktischen Inversion (Freudenthal) seit der Jahrtausendwende die Kompetenz- und Output-Orientierung zu einer No Math-Bewegung geführt hat, die durch anti-didaktische Omission gekennzeichnet ist. Wenn sich der schulische Mathematikunterricht weiterhin zu einer Parallelkultur entwickelt, die sich immer weiter von der Jahrtausende alten Kultur der Mathematik ablöst, werden die Studierenden der Mathematik oder verwandter Studiengänge nicht nur mathematisch noch schwächer, sondern sie werden ausbleiben, wie wir das in den Niederlanden vor etwa zehn Jahren in extremer Form (Kaenders 2009) gesehen haben. Es muss dringend etwas geschehen, wenn wir die Beschädigung der mathematischen Kultur begrenzen wollen.

In ihrer Stellungnahme [4] „Zur aktuellen Diskussion über die Qualität des Mathematikunterrichts“ vom 20. 4. 2017 erkennt die Mathematik-Kommission Übergang Schule-Hochschule der Verbände DMV, GDM und MNU erstmalig die Krise des Mathematikunterrichts in aller Deutlichkeit an:

An deutschen Hochschulen verzeichnet man seit mehr als einer Dekade den alarmierenden Befund, dass einem Großteil der Studierenden bei Studienbeginn viele mathematische Grundkenntnisse und -fertigkeiten sowie konzeptuelles Verständnis mathematischer Inhalte fehlen.

Die Kommission weist die Verantwortung der Reduktion des Mathematikunterrichts in den unterschiedlichen Stundentafeln der Länder, dem Wegfall oder der Veränderung des Leistungskurssystems, dem hohen Anteil von Abiturienten in jedem Jahrgang, dem Spannungsfeld zwischen Allgemeinbildung und der Vorbereitung auf mathematikhaltige Studiengänge und der Einführung neuer Inhalte zu. Seit ihrem Bestehen hat die Kommission die wichtige Aufgabe übernommen, die Verbesserung dieser Rahmenbedingungen bei der Politik einzufordern [5].

Die Kommission empfiehlt jedoch im Namen der Fachgesellschaften auch, weiterhin und noch mehr auf die kompetenzorientierten Bildungsstandards zu setzen. Es sei nicht zu bestreiten, dass mit den Bildungsstandards der richtige bildungspolitische Weg eingeschlagen wurde, der es ermöglicht, bundesweit bislang stark unterschiedliche Bestimmungen besser aufeinander abzustimmen. Nur sollen diese jetzt noch verbindlicher und noch konkreter formuliert werden.

Wir denken, diese Schlussfolgerung der Kommission sollte unbedingt erst in einem fundierten theoretischen Diskurs überdacht werden, bevor der vorgeschlagene Weg der Kompetenzorientierung weiter beschritten wird. Sollte der Appell der Kommission an die Politik bezüglich der Rahmenbedingungen für Mathematikunterricht Gehör finden, bedeutet dies ansonsten: In Zukunft werden bundeseinheitlich in mehr Stunden pro Woche, teilweise in Leistungskursen, mit etwas weniger Gymnasiasten als jetzt zur Vorbereitung auf ein mathematikhaltiges Studium noch intensiver als bisher Schneehöhen mit DFunktionen modelliert und das Ganze wird von Psychologen am IQB in seiner Qualität gesichert.

Die Autoren danken Wolfgang Kühnel für konstruktive und hilfreiche Anmerkungen zu diesem Beitrag.

\section{Literatur}

Bandelt, J. \& Kühnel, W. (2016): Schöne neue Mathewelt. Mitteilungen der GDM, 100, 30-32.

Bandelt, J. \& Kühnel, W. (2017): Schöne neue Mathewelt. Mitteilungen der GDM, 102, 16-18.

Baumert, J., Klieme, E., Neubrand, M., Prenzel, M., Schiefele, U., Schneider, W., Tillmann, K.-J. \& Weiß, M. (200o): Die Fähigkeit zum Selbstregulierten Lernen als fächerübergreifende Kompetenz. Berlin: Max-Planck-Institut für Bildungsforschung.

Blum, W., Drüke-Noe, C., Hartung, R. \& Köller, O. (Hrsg.) (2006): Vorwort der Herausgeber. Bildungsstandards Mathematik: konkret Sekundarstufe I: Aufgabenbeispiele, Unterrichtsanregungen, Fortbildungsideen. IQB: Cornelsen Verlag Scriptor, Berlin.

Blum, W. (2016): Leserbrief zu: Franz Lemmermeyer: Abituraufgaben und Kompetenz. Mitteilungen der DMV 24-4, S. 188.

Dörpinghaus, A. (2009): Bildung: Plädoyer wider die Verdummung. Forschung \& Lehre 9/2009, Supplement.

Gelhard, A. (2012): Kritik der Kompetenz. Diaphanes. Zürich.

Greefrath, W., Ludwig, M. \& Siller, S. (2016): Leserbrief zu: Franz Lemmermeyer: Abituraufgaben und Kompetenz. Mitteilungen der $D M V$ 24-4, 188-189.

Jahnke, Th. (2016): Anwendungsorientierter Mathematikunterricht ein didaktisches Missverständnis? Vortragsausarbeitung Toeplitz Kolloquium, HCM Bonn am 14. Juni 2016.

Kaenders, R. (2009): Von Wiskunde und Windmühlen - über den Mathematikunterricht in den Niederlanden. Beiträge zum Mathematikunterricht, Hauptvortrag GDM-Tagung in Oldenburg, GDM, WTM-Verlag Münster. www.mathematik.tu-dortmund.de/ ieem/cms/media/BzMU/BzMU20og/Beitraege/Hauptvortraege/ KAENDERS_Rainer_2009_Windmuehlen.pdf

Klein, H.-P. und Jahnke, T. (2012): Die Folgen der Kompetenzorientierung im Fach Mathematik. Journal für Didaktik der Biowissenschaften (F) 2, 1-9 (2012)

Klein, H.-P. (2016): Vom Streifenhörnchen zum Nadelstreifen - Das deutsche Bildungswesen im Kompetenztaumel. Zu Klampen Verlag.

Lemmermeyer, F. (2016): Abituraufgaben und Kompetenz, Mitteilungen der DMV 24-3, 170-173.

Ryan, R. M. \& Deci, E. L. (200o): Intrinsic and Extrinsic Motivations: Classic Definitions and New Directions. Contemporary Educational Psychology 25, 54-67. 
Weinert, F. E. (Hrsg.) (2002): Leistungsmessungen in Schulen. Weinheim/Basel: Beltz Verlag.

Wiechmann, R. (2013): Zur Verabsolutierung des Problemlösens im Kompetenzkonzept und ihren Folgen. Vierteljahresschrift für wissenschaftliche Pädagogik 1/2013, 124-147.
Internetquellen

[1] https://tinyurl.com/y7hoozx5 (24.4.2017)

[2] https://tinyurl.com/ybwmklab (24.4.2017)

[3] https://tinyurl.com/yd 7 pvvpr (24.4.2017)

[4] https://tinyurl.com/yaslmdzf (24. 4. 2017)

[5] https://tinyurl.com/yc3y4u94 (27.4.2017)

Ysette Weiss forscht und lehrt als Mathematikdidaktikerin am Institut für Mathematik der Johannes Gutenberg-Universität Mainz.

Rainer Kaenders forscht und lehrt in ,Mathematik und ihrer Didaktik' am Mathematischen Institut wie auch im Hausdorff-Zentrum für Mathematik an der Rheinischen Friedrich-Wilhelms-Universität Bonn.

Beide haben in Mathematik promoviert und verfügen jeweils über mehrjährige Erfahrungen in verschiedenen Bildungssystemen. Weiss in der DDR, in der Sowjetunion, in England und schließlich im vereinigten Deutschland. Kaenders in der BRD, in den Niederlanden und in demselben vereinigten Deutschland.

Prof. Dr. Ysette Weiss, Institut für Mathematik, Johannes Gutenberg-Universität Mainz Staudingerweg 9, 55128 Mainz weisspid@uni-mainz.de

Prof. Dr. Rainer Kaenders, Mathematisches Institut, Hausdorff Center for Mathematics,

Rheinische Friedrich-Wilhelms-Universität Bonn, Endenicher Allee 6o, 53115 Bonn r.kaenders@uni-bonn.de

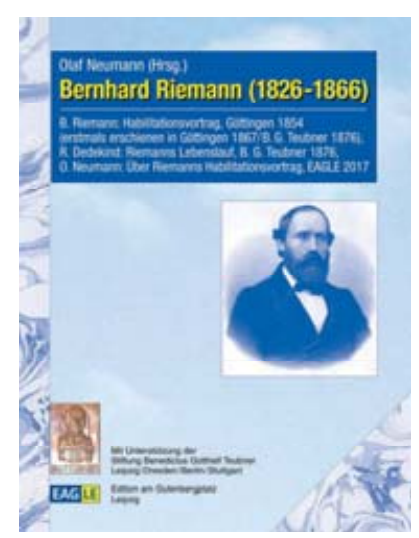

O. Neumann (Hrsg.). EAGLE 097. 1. A. 2017. HC. 978-3-95922-097-2

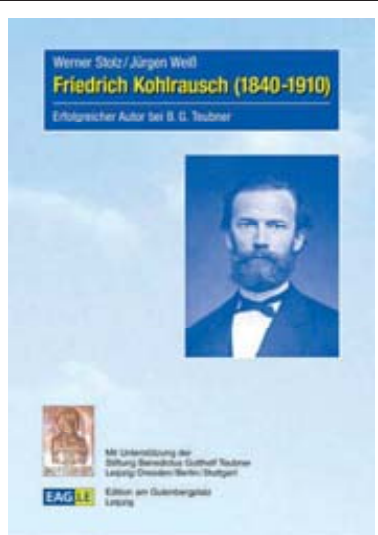

W. Stolz / J. Weiß. EAGLE 095. 1. A. 2017. HC. $978-3-95922-095-8$

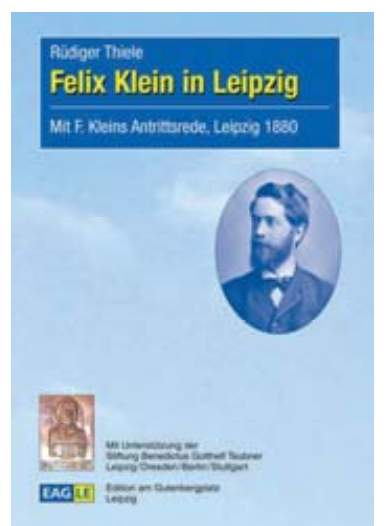

R. Thiele. EAGLE 047.

1. A. 2011. 978-3-937219-47-9

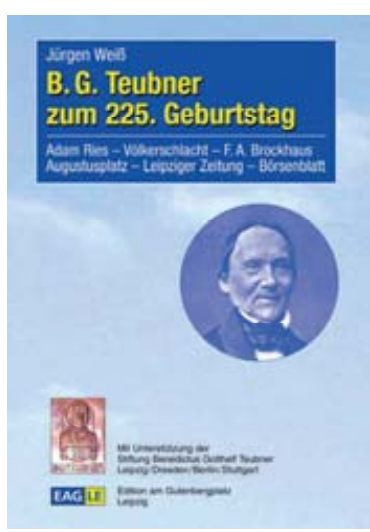

J. Weiß. EAGLE 035.

1. A. 2009. 978-3-937219-35-6

Edition am Gutenbergplatz Leipzig: www.eagle-leipzig.de I www.eagle-leipzig.de/starthilfen.htm I https://twitter.com/EagleLeipzig NEU/April 2017: Olaf Neumann, Bernhard Riemann (1826-1866). EAGLE 097. B. Riemanns Habilitationsvortrag erschien erstmals vor 150 Jahren, in Göttingen 1867. H. Weyl (1919): "... daß diese Schrift, ein bewunderungswürdiges Meisterstück, in möglichst viele Hände kommt."

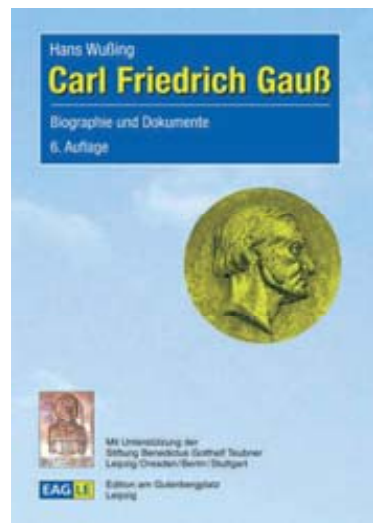

H. Wußing. EAGLE 051. 6. A. 2011. 978-3-937219-51-6

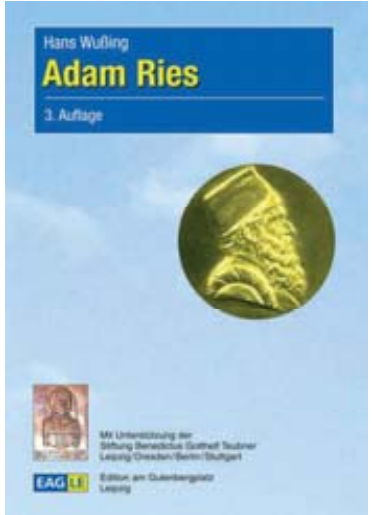

H. Wußing. EAGLE 033. 3. A. 2009. 978-3-937219-33-2

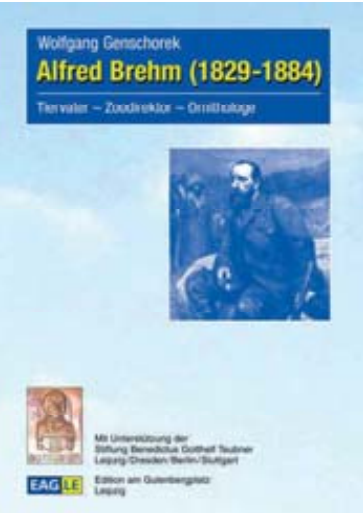

W. Genschorek. EAGLE 092. 1. A. 2016. 978-3-95922-092-7

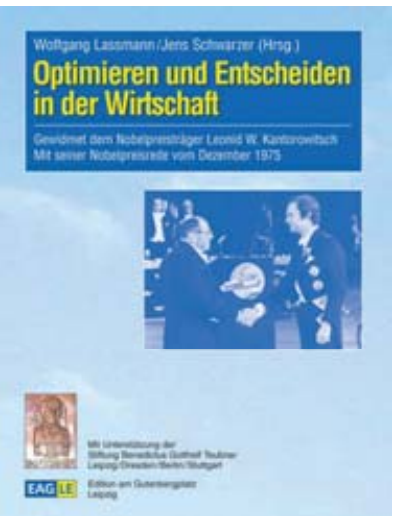

W. Lassmann/J. Schwarzer. EAGLE 013. 1. A. 2004. 3-937219-13-7 